\title{
Influence of Post Emergence Application of K Salt of Glyphosate on Weed Control and Yield of Transgenic Stacked and Non Transgenic Maize Hybrids in Summer Irrigated Eco systems of Tamil Nadu, India
}

\author{
K. Sivagamy ${ }^{1 *}$, C. Chinnusamy ${ }^{2}$ and P. Parasuraman ${ }^{1}$ \\ ${ }^{1}$ Centre of Excellence for Millets, Athiyandal, Thiruvannamalai -606 603, India \\ ${ }^{2}$ AICRP on Weed control, Tamil Nadu Agricultural University, Coimbatore-641 003, India
}

*Corresponding author

\begin{tabular}{|c|}
\hline Keywords \\
\hline $\begin{array}{l}\text { Herbicide tolerant } \\
\text { maize, Absolute and } \\
\text { relative density, } \\
\text { Weed control } \\
\text { efficiency and Yield }\end{array}$ \\
\hline Article Info \\
\hline $\begin{array}{l}\text { Accepted: } \\
\text { 10 December } 2018 \\
\text { Available Online: } \\
\text { 10 January } 2019\end{array}$ \\
\hline
\end{tabular}

\section{A B S T R A C T}

A field investigation was carried out at Tamil Nadu Agricultural University, Coimbatore during kharif seasons of 2010 and 2011 to evaluate the weed control options for transgenic stacked (TC 1507 and NK 603) and non-transgenic maize hybrids. The experiments were conducted with the following objective to evaluate the weed control efficiency and crop productivity with $\mathrm{K}$ salt of glyphosate formulations under field conditions. Treatments consisted of two transgenic stacked hybrids named 30V92 and 30B11 applied with glyphosate as early post emergence at 900 and 1800 ga.e ha $^{-1}$ during kharif, 2010 and conventional maize hybrids named 30V92 and 30B11 applied with glyphosate by controlled droplet application method at 900, 1350 and 1800 ga.e ha ${ }^{-1}$ during kharif,2011compared with non-transgenic counterpart maize hybrids applied with pre emergence atrazine at $0.5 \mathrm{~kg} \mathrm{ha}^{-1}$ followed by one hand weeding on 40 DAS with and without insect management. Among the treatments, Early POE application of glyphosate at 1800 ga.e ha $^{-1}$ registered lower weed density and higher weed control efficiency in transgenic and non-transgenic maize hybrids at all the intervals. Higher grain yield was registered with post emergence application of glyphosate at 1800 ga.e ha $^{-1}$ in transgenic and non transgenic maize hybrid of 30V92 during both the seasons of study.

\section{Introduction}

Maize (Zea mays L.) is an important cereal in many developed and developing countries of the world and provides maximum share of human food. Maize has derived its name from the word "mahis" meaning source of life. Maize provides nutrients for humans and animals and serves as a basic raw material for the production of starch, oil, protein, alcoholic beverages, food sweeteners and more recently fuel maize accounts for over $30 \%$ of global cereal output and still the demand and production of maize have been increasing continuously. There is no cereal crop on the earth that has so much yield potential and hence it is popularly called "Queen of Cereals". The major yield reducing factors for maize cultivation in India are weeds and insects. Weeds cause considerable yield loss 
due to competition of resources with maize crop was stated by Malviya and Singh (2007). The development of crop cultivar with resistance to selected herbicides has the positive impact on agricultural production. Selection of proper herbicides is essential for successful weed management in all crop production systems.

Post emergence herbicides have been achieved adequate weed control programmes, due to its broad spectrum of activity, excellent crop safety, convenience and flexibility was reported by Ferrel and Witt (2002). This necessitates the development and testing of selective early post emergence herbicides for weed control in maize. Glyphosate is a foliar applied, broad spectrum, post emergence herbicide capable of controlling annual, perennial grasses and dicotyledonous weeds. The introduction of glyphosate resistant crops has created new opportunities for the use of effective, non selective herbicides like glyphosate as selective weed control in crop production. Prior to the introduction of glyphosates resistant crops, glyphosate is being applied to control existing vegetation prior to sowing the crops. Advancement in biotechnological research enables genetic engineering to enhance production and minimize crop yield losses through development of crops that are tolerant to insects and herbicides.

Genetically modified crops are the most rapidly adopted technology in agricultural history due to the social and economic benefits. Now, it can be used as post emergence herbicide in glyphosate resistant crops (Norsworthy, 2001). A transgenic stacked maize (TC1507 x NK603) obtained by crossing two transgenic maize lines by using traditional plant breeding techniques. TC1507 maize expresses a $\mathrm{Bt}$ insecticidal protein (Cry1F) for control of certain lepidopteron (stem borers) pests, and NK603 corn expresses a modified maize 5enolpyruvylshikimate-3-phosphate synthase enzyme (CP4 EPSPS) that confers tolerance to herbicide products containing glyphosate. The CP4EPSPS proteins have a low affinity for glyphosate compared to the wild-type EPSPS enzyme. Thus, when corn plants expressing the CP4 EPSPS proteins are treated with glyphosate, the plants continue to grow.

Post emergence application of glyphosate at $1800 \mathrm{~g}$ a.e ha ${ }^{-1}$ gave significantly lower weed index, weed dry weight and high weed control efficiency at all the intervals. Post emergence application of glyphosate at $1800 \mathrm{~g}$ a.e ha ${ }^{-1}$ in transgenic maize and post emergence control droplet application method of glyphosate at $1800 \mathrm{~g}$ a.e ha ${ }^{-1}$ in conventional maize hybrid (30V92) recorded high productivity and profitability. In view of the above facts, an experiment on "Influence of Post Emergence Application of K Salt of Glyphosate on Weed Control and Yield of Transgenic Stacked and Non Transgenic Maize taken up during summer season of 2010 and 2011

\section{Materials and Methods}

The field experiments were conducted during kharif seasons of 2010 and 2011 in Eastern block farm of Tamil Nadu Agricultural University at Coimbatore. The soil of the experimental site was sandy clay loam in texture $(32.48 \%$ clay, $18.50 \%$ silt and 28.96 $\%$ coarse sand) with low available nitrogen, medium in available phosphorous and high in available potassium. Treatment details are given in Tables.

The experiment was laid in Randomized Block Design (RBD) with three replications. The adopted spacing between the rows and plants were $60 \mathrm{~cm}$ and $25 \mathrm{~cm}$ respectively. Herbicide tolerant transgenic maize test hybrids namely 30V92, 30B11 and conventional hybrids of 30V92, 30B11, BIO 
9681 and COHM5 during kharif, 2010. POE application of glyphosate was done at 25 DAS of transgenic and non-transgenic maize. Conventional maize hybrids 30V92, 30B11, BIO 9681 and COHM5 were raised during kharif, 2011. Observations were made on predominant weed flora, weed control efficiency and maize yield. The herbicides as per the treatments schedule were applied as pre emergence at third day after sowing, glyphosate application at 2-4 leaf stage of weeds (20-25 DAS of maize). Hand operated knapsack sprayer fitted with a flat fan type nozzle (WFN 40) was used for spraying the herbicides adopting a spray volume of 250 litres $\mathrm{ha}^{-1}$.The recommended dose of 150:75:75 Kg of NPK ha ${ }^{-1}$ in the form of urea, single super phosphate and muriate of potash. During the course of experiment, the data were revealed our predominant weed flora, weed density and dry weight with grain yield.

\section{Results and Discussion}

\section{Effect on weeds}

\section{Predominant weed flora of the experimental field}

Weed flora of the experimental field in maize was predominantly consisted of twelve species of broad leaved weeds, five species of grasses and a sedge weed. The dominant among broadleaved weeds were Trianthem aportulacastrum, Datura stramonium, Cleome gynandra, Digera arvensis, Physallis minima and Corchorus olitorius. The dominant grass weeds were Setaria verticillata and Cynodon dactylon. Cyperus rotundus was the only sedge present in the experimental field.

The different treatments had significant influence on weed flora. During kharif2010, before herbicide spray, broad leaved weeds dominated the weed flora recording 86.09 per cent and it was followed by grasses 5.41 per cent and sedge 8.48 per cent. With regard to the dominance of weed species during kharif, 2011 before first spraying of glyphosate, broad leaved weeds dominated recording 83.27 per cent and this was followed by grasses 9.14 per cent and sedge 7.59 per cent (Table 1).

With respect to individual weed species during both the years, density of Trianthem aportulacastrum recorded about 162.80 No. $\mathrm{m}^{-2}$ per cent before spraying of glyphosate. Higher weed flora composition registered during both the years might be due to adequate rainfall during cropping period favoured a conducive field environment for weed growth.

Trianthem aportulacastrum, Datura stramonium, Cleome gynandra, Physallis minima, Digera arvensis, Setaria verticillata and Cynodondactylon. The results are in line with the findings of (Nadeem et al., 2008) who have reported that Trianthem aportulacastrum, Digera arvens is were the most common weeds which compete with maize and assimilate faster biomass than maize.

\section{Weed density}

The weed control methods effectively controlled the density of all the weeds under both transgenic and non-transgenic maize hybrids at different stages of crop growth as compared to unweeded control (Table 1). During kharif 2010, lower weed density was achieved under non transgenic maize hybrid BIO 9681 and 30B11 with pre emergence application of atrazine at $0.5 \mathrm{Kg} \mathrm{ha}^{-1}$ followed by hand weeding at 20 DAS. Relatively, a higher density was observed under unweeded checks and transgenic maize before imposing post emergence application of glyphosate. Atrazine effectively controlled majority of broad leaved and grassy weeds at earlier stages of maize growth. Mundra et al., (2003) reported that application of atrazine at $0.5 \mathrm{~kg}$ 
$\mathrm{ha}^{-1}$ as pre-emergence $f b$ inter cultivation at 35 DAS in maize significantly reduced the total weed density.

At 40 DAS and 60 DAS, lower weed density (2.04 and 2.35) was observed under transgenic maize hybrid 30V92 with post emergence application of glyphosate at $1800 \mathrm{~g}$ a.e ha ${ }^{-1}$ resulted in effective control of broad leaved weeds, grasses and sedges due to its broad spectrum action (Wilcut et al., 1996). This may due to more impressive control of broadleaved weeds like Trainthem aportulacastrum, Datura stramonium, Cleome gynandra and Physallis minima. Foliar application of glyphosate was readily and rapidly translocated throughout the actively growing aerial and under ground portions at active growing stage of broadleaved weeds might have blocked the 5-Enulpyruvate shikimate -3- phosphate synthase enzyme and arrest the amino acid synthesis which led to complete control (Summons et al., 1995) (Table 2).

During kharif 2011, post emergence controlled droplet application of glyphosate at conventional maize hybrid of $30 \mathrm{~V} 92$ at $1800 \mathrm{~g}$ a.e $\mathrm{ha}^{-1}\left(1.84 \mathrm{Nos} \mathrm{m}^{-2}\right)$ observed lesser total weed density at 40 DAS. Thus glyphosate effectively controlled a broad spectrum of annual and perennial grasses, sedges and broadleaved weeds could be due to increased translocation of glyphosate inside the plant tissues Suwunnamek and Parker (1975) (Table 3).

\section{Effect on crop}

A broad spectrum of weeds with wider adaptability to extremities of climatic, edaphic and biotic stresses is infesting the maize fields. High persistence nature of weeds was attributed to their ability of high seed production and seed viability. Post emergence herbicides have been achieved adequate weed control programmes.
During both the years of study, among the weed control treatments, post emergence application of glyphosate at $1800 \mathrm{~g}$ a.e $\mathrm{ha}^{-1}$ in transgenic corn hybrid recorded higher grain yield of $12.21 \mathrm{t} \mathrm{ha}^{-1}$ this was 36.64 percent higher than the unweeded check plot of transgenic 30V92 during kharif, 2010 (Table 4). Whereas during kharif 2011, post emergence controlled droplet application of glyphosate at $1800 \mathrm{~g}$ a.e $\mathrm{ha}^{-1}$ in conventional maize hybrid of $30 \mathrm{~V} 92$ resulted in higher grain yield of $11.23 \mathrm{t} \mathrm{ha}^{-1}$ (Table 5). This was 44.79 percent higher than the unweeded check plot of conventional maize hybrid. This could be achieved control of weeds with non selective, translocated herbicide, provided the favourable crop growth environment at the establishment stage of the crop itself by minimizing the perennial and annual weeds and increased the seed and stalk yields (Tharp et al.,1999).This might be due to the fact that the perennial weeds like Cyperus rotundus, Cynodon dactylon, troublesome broadleaved weeds like Trianthem aportulacastrum weeds were effectively controlled and might increase the maize yield may be due to better light utilization of narrow row zone and faster canopy closure (Murphy et al., 1999).

Maize grain yield of $\mathrm{POE}$ application of glyphosate at $1800 \mathrm{~g}$ a.e $\mathrm{ha}^{-1}$ in transgenic $30 \mathrm{~V} 92\left(\mathrm{~T}_{2}\right)$ was taken as basis to work out the weed index (WI) during kharif, 2010. In transgenic maize hybrids, among the different rates of glyphosate, $900 \mathrm{~g}$ a.e $\mathrm{ha}^{-1}$ recorded lower weed index of (9.09 and 10.15 per cent) in transgenic 30V92 $\left(\mathrm{T}_{1}\right)$ and 30B11 $\left(\mathrm{T}_{4}\right)$ respectively. In non-transgenic maize hybrids, $\mathrm{PE}$ application of atrazine $0.5 \mathrm{~kg} \mathrm{ha}^{-1}+\mathrm{HW}$ in 30V92 recorded lesser weed index (16.21per cent) compared all other non-transgenic hybrids with same treatment. During kharif2011 among the different rates of glyphosate by controlled droplet application method of glyphosate at $1350 \mathrm{~g}$ a.e $\mathrm{ha}^{-1}$ recorded lower weed index of 7.75 and 15.23 
per cent in non transgenic maize hybrids of 30V92 $\left(\mathrm{T}_{2}\right)$ and 30B11( $\left.\mathrm{T}_{5}\right)$. It was followed by POE application of glyphosate at $900 \mathrm{~g}$ a.e $\mathrm{ha}^{-1}$ in both non transgenic maize hybrids viz., 30V92 and 30B11. However, in PE application of atrazine at $0.5 \mathrm{~kg} \mathrm{ha}^{-1} \mathrm{fb} \mathrm{HW}$ in $30 \mathrm{~V} 92\left(\mathrm{~T}_{7}\right)$ maize hybrid recorded least weed index compared all other non-transgenic hybrids with same treatment. Unweeded check plots resulted in higher weed index and performed poorly during both the years. Among the weed control treatments methods, lower weed index was recorded with 9.09 and 10.15 per cent in transgenic $30 \mathrm{~V} 92\left(\mathrm{~T}_{1}\right)$ and 30B11( $\left.\mathrm{T}_{5}\right)$ whereas at conventional maize hybrids observed glyphosate at $1350 \mathrm{~g}$ a.e ha ${ }^{-1}$ recorded lower weed index of 7.75 and 15.23 per cent in non transgenic maize hybrids of 30V92 $\left(\mathrm{T}_{2}\right)$ and 30B11( $\left.\mathrm{T}_{5}\right)$. Unweeded check plots resulted in higher weed index and performed poorly during both the years of study. Weed control efficiency which indicates the comparative magnitude of reduction in weed dry matter, was highly influenced by different weed control treatments. Pre emergence application of atrazine at $0.5 \mathrm{Kg} \mathrm{ha}{ }^{-1}$ followed by hand weeding recorded higher weed control efficiency of 80.28 percent in non transgenic maize hybrid $30 \mathrm{~V} 92$ at 20 DAS. Whereas at 40 DAS after spraying of herbicide, higher weed control efficiency of 99.53 per cent was recorded in glyphosate at $1800 \mathrm{~g}$ a.e $\mathrm{ha}^{-1}$ followed by 30B11was observed 98.97 per cent during kharif,2010 (Table 4). Whereas during kharif, 2011 higher weed control efficiency was observed with glyphosate at $1800 \mathrm{~g}$ a.e $\mathrm{ha}^{-1}$ in conventional maize hybrid of 30V92 registered maximum weed control efficiency of 99.14 per cent owing to the fact that registered lesser weed density (Table 5).

Table.1 Absolute density (AD) and Relative density (RD) of predominant weed species in transgenic and non transgenic maize before first herbicide spray (20 DAS)

\begin{tabular}{|c|c|c|c|c|}
\hline \multirow[t]{3}{*}{ Weeds } & \multicolumn{2}{|c|}{ Kharif, 2010} & \multicolumn{2}{|c|}{ Kharif, 2011} \\
\hline & \multicolumn{2}{|c|}{ AD $\quad$ RD } & \multicolumn{2}{|c|}{ AD $\quad$ RD } \\
\hline & $\left(\mathrm{No} . / \mathrm{m}^{2}\right)$ & $(\%)$ & $\left(\mathrm{No} . / \mathrm{m}^{2}\right)$ & $(\%)$ \\
\hline \multicolumn{5}{|l|}{ Broad leaved weeds } \\
\hline Trianthem aportulacastrum & 162.80 & 57.57 & 162.3 & 49.69 \\
\hline Datura stramonium & 20.80 & 7.36 & 22.00 & 6.74 \\
\hline Cleome gynandra & 29.67 & 10.49 & 30.67 & 9.39 \\
\hline Digera arvensis & 10.20 & 3.61 & 27.66 & 8.47 \\
\hline Physalis minima & 8.00 & 2.83 & 16.35 & 5.01 \\
\hline Other BLW & 12.00 & 4.24 & 13.00 & 3.98 \\
\hline Total BLW & 243.47 & 86.09 & 271.98 & 83.27 \\
\hline \multicolumn{5}{|l|}{ Grasses } \\
\hline Cynodon dactylon & 6.00 & 2.12 & 19.25 & 5.89 \\
\hline Setaria verticillata & 8.12 & 2.87 & 9.30 & 2.85 \\
\hline Other grasses & 1.20 & 0.42 & 1.30 & 0.40 \\
\hline Total grasses & 15.32 & 5.41 & 29.85 & 9.14 \\
\hline \multicolumn{5}{|l|}{ Sedge } \\
\hline Cyperus rotundus & 24.00 & 8.48 & 24.80 & 7.59 \\
\hline Total weed density & 282.79 & 100.00 & 326.63 & 100.00 \\
\hline
\end{tabular}

Data not statistically analysed 
Table.2 Effect of glyphosate application on total weed density in transgenic maize

\begin{tabular}{|c|c|c|c|}
\hline \multirow[t]{3}{*}{ Treatments } & \multicolumn{3}{|c|}{ Total weed density (No. $\mathrm{m}^{-2}$ ) } \\
\hline & \multicolumn{3}{|c|}{ Kharif, 2010} \\
\hline & 20 DAS & 40 DAS & 60 DAS \\
\hline $\begin{array}{l}\text { T } 1 \text { - T.30V92 HR Glyphosate @ } 900 \text { g a.e } \\
\text { ha }^{-1}\end{array}$ & $15.43(236.22)$ & $\begin{array}{l}2.78 \\
(5.75)\end{array}$ & $\begin{array}{l}3.41 \\
(9.63)\end{array}$ \\
\hline $\begin{array}{l}\text { T2-T.30V92HR Glyphosate @ } 1800 \text { g a.e } \\
\text { ha }^{-1}\end{array}$ & $15.33(233.08)$ & $\begin{array}{l}2.04 \\
(2.15)\end{array}$ & $\begin{array}{l}2.35 \\
(3.52)\end{array}$ \\
\hline $\mathrm{T}_{3}-\mathrm{T} .30 \mathrm{~V} 92 \mathrm{HR}$ (Weedy check) & $15.74(245.60)$ & $\begin{array}{l}14.32 \\
(202.93)\end{array}$ & $\begin{array}{l}13.81 \\
(188.75)\end{array}$ \\
\hline $\begin{array}{l}\text { T} 4-\text { T.30B11HR Glyphosate @900 g a.e } \\
\text { ha }^{-1}\end{array}$ & $15.78(246.89)$ & $\begin{array}{l}3.31 \\
(8.98)\end{array}$ & $\begin{array}{l}3.84 \\
(12.74)\end{array}$ \\
\hline $\begin{array}{l}\text { T5 } \\
\text {-T.30B11HR Glyphosate @1800 g a.e } \\
\text { ha }^{-1}\end{array}$ & $16.06(256.07)$ & $\begin{array}{l}2.55 \\
(4.50)\end{array}$ & $\begin{array}{l}3.06 \\
(7.35)\end{array}$ \\
\hline $\mathrm{T}_{6}-\mathrm{T} .30 \mathrm{~B} 11 \mathrm{HR}$ (Weedy check) & $15.81(248.10)$ & $\begin{array}{l}14.54 \\
(209.43)\end{array}$ & $\begin{array}{l}14.42 \\
(205.99)\end{array}$ \\
\hline $\begin{array}{l}\mathrm{T}_{7}-\mathrm{N} . \mathrm{T} .30 \mathrm{~V} 92 \mathrm{PE} \text { atrazine } 0.5 \mathrm{~kg} \mathrm{ha}^{-1}+ \\
\text { HW+ IC }\end{array}$ & $\begin{array}{l}7.99 \\
(61.85)\end{array}$ & $\begin{array}{l}7.81 \\
(59.00)\end{array}$ & $\begin{array}{l}5.79 \\
(31.48)\end{array}$ \\
\hline $\mathrm{T}_{8}-$ N.T.30V92 No WC and only IC & $15.45(236.55)$ & $\begin{array}{l}13.64 \\
(183.99\end{array}$ & $\begin{array}{l}12.74 \\
(160.36)\end{array}$ \\
\hline $\mathrm{T}_{9}-$ N.T.30V92 No WC and no IC & $16.05(255.75)$ & $\begin{array}{l}14.37 \\
(204.37)\end{array}$ & $\begin{array}{l}14.38 \\
(204.69)\end{array}$ \\
\hline $\begin{array}{l}\mathrm{T}_{10^{-}} \text {N.T.30B11 PE atrazine } 0.5 \mathrm{~kg} \mathrm{ha}^{-1}+ \\
\text { HW+ IC }\end{array}$ & $\begin{array}{l}7.55 \\
(55.00)\end{array}$ & $\begin{array}{l}8.14 \\
(64.34)\end{array}$ & $\begin{array}{l}5.87 \\
(32.43)\end{array}$ \\
\hline $\mathrm{T}_{11}-$ N.T.30B11No WC and only IC & $15.51(238.44)$ & $\begin{array}{l}13.58 \\
(182.38)\end{array}$ & $\begin{array}{l}13.12 \\
(170.11)\end{array}$ \\
\hline $\mathrm{T}_{12}-$ N.T.30B11 No WC and no IC & $16.25(262.00)$ & $\begin{array}{l}15.05 \\
(224.47)\end{array}$ & $\begin{array}{l}15.05 \\
(224.57)\end{array}$ \\
\hline $\begin{array}{l}\mathrm{T}_{13}-\mathrm{BIO} 9681 \mathrm{PE} \text { atrazine } 0.5 \mathrm{~kg} \text { ha } \\
{ }^{-}+\mathrm{HW}+\mathrm{IC}\end{array}$ & $\begin{array}{l}7.15 \\
(49.14)\end{array}$ & $\begin{array}{l}7.52 \\
(54.58)\end{array}$ & $\begin{array}{l}5.96 \\
(33.49)\end{array}$ \\
\hline $\mathrm{T}_{14}$-BIO9681No WC and no IC & $14.69(213.70)$ & $\begin{array}{l}13.85 \\
(189.93)\end{array}$ & $\begin{array}{l}14.52 \\
(208.94)\end{array}$ \\
\hline $\begin{array}{l}\mathrm{T}_{15}-\mathrm{CoHM} 5 \mathrm{PE} \text { atrazine } 0.5 \mathrm{~kg} \mathrm{ha}^{-} \\
+\mathrm{HW}+\mathrm{IC}\end{array}$ & $\begin{array}{l}7.83 \\
(59.37)\end{array}$ & $\begin{array}{l}8.32 \\
(67.3)\end{array}$ & $\begin{array}{l}6.20 \\
(36.44)\end{array}$ \\
\hline $\mathrm{T}_{16}-\mathrm{CoHM} 5$ No WC and no IC & $16.38(266.19)$ & $\begin{array}{l}15.24 \\
(230.37)\end{array}$ & $\begin{array}{l}15.79 \\
(247.44)\end{array}$ \\
\hline SEd & 1.34 & 1.11 & 1.06 \\
\hline $\mathrm{CD}(\mathbf{P}=\mathbf{0 . 0 5})$ & 2.74 & 2.27 & 2.17 \\
\hline $30 \mathrm{~V} 92$ & 92-Nor & enic & $92, T .30 \mathrm{~B} 11$ \\
\hline
\end{tabular}

Transgenic30B11,N.T.30B11-Non transgenic 30B11;HW-Hand weeding; IC-Insect control; WC-Weed control 
Table.3 Effect of glyphosate application on total weed density in nontransgenic maize

\begin{tabular}{|c|c|c|c|}
\hline \multirow[t]{3}{*}{ Treatments } & \multirow{2}{*}{\multicolumn{3}{|c|}{ Total weed density $\left(\right.$ No. $\left.\mathrm{m}^{-2}\right)$}} \\
\hline & & & \\
\hline & 20 DAS & 40 DAS & 60 DAS \\
\hline 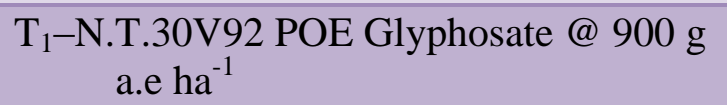 & $16.61(273.97)$ & $\begin{array}{l}4.11 \\
(14.89)\end{array}$ & $\begin{array}{l}4.61 \\
(19.29)\end{array}$ \\
\hline $\begin{array}{l}\text { T2- N.T.30V92 POE Glyphosate @ } 1350 \\
\text { g a.e ha }^{-1}\end{array}$ & $16.25(262.05)$ & $\begin{array}{l}2.91 \\
(6.45)\end{array}$ & $\begin{array}{l}3.69 \\
(11.62)\end{array}$ \\
\hline $\begin{array}{l}\text { T3-N.T.30V92 POE Glyphosate @ } 1800 \\
\text { g a.e ha }^{-1}\end{array}$ & $16.52(271.05)$ & $\begin{array}{l}1.84 \\
(1.4)\end{array}$ & $\begin{array}{l}2.85 \\
(6.10)\end{array}$ \\
\hline $\begin{array}{l}\mathrm{T}_{4}-\text { N.T.30B11 POE Glyphosate @ } 900 \mathrm{~g} \\
\text { a.e ha- }\end{array}$ & $16.41(267.29)$ & $\begin{array}{l}4.32 \\
(16.65)\end{array}$ & $\begin{array}{l}4.84 \\
(21.41)\end{array}$ \\
\hline $\begin{array}{l}\mathrm{T}_{5} \text {-N.T.30B11 POE Glyphosate @ } 1350 \mathrm{~g} \\
\text { a.e ha }\end{array}$ & $16.60(273.46)$ & $\begin{array}{l}3.16 \\
(8.01)\end{array}$ & $\begin{array}{l}4.16 \\
(15.27)\end{array}$ \\
\hline 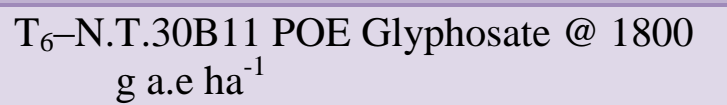 & $16.93(284.57)$ & $\begin{array}{l}2.23 \\
(2.99)\end{array}$ & $\begin{array}{l}3.36 \\
(9.32)\end{array}$ \\
\hline $\begin{array}{l}\mathrm{T}_{7}-30 \mathrm{~V} 92 \mathrm{PE} \text { atrazine } 0.5 \mathrm{~kg} \mathrm{ha}^{-1}+ \\
\text { HW+ IC }\end{array}$ & $\begin{array}{l}7.37 \\
(52.27)\end{array}$ & $\begin{array}{l}8.78 \\
(75.16)\end{array}$ & $\begin{array}{l}6.81 \\
(44.43)\end{array}$ \\
\hline $\mathrm{T}_{8}-30 \mathrm{~V} 92 \mathrm{No} \mathrm{WC}$ and only IC & $16.35(265.46)$ & $\begin{array}{l}14.83 \\
(217.99)\end{array}$ & $\begin{array}{l}14.58 \\
(210.68)\end{array}$ \\
\hline $\mathrm{T}_{9}-30 \mathrm{~V} 92 \mathrm{No} \mathrm{WC}$ and no IC & 17.03 & $\begin{array}{l}15.49 \\
(238.01)\end{array}$ & $\begin{array}{l}15.35 \\
(233.48)\end{array}$ \\
\hline $\begin{array}{l}\mathrm{T}_{10}-30 \mathrm{~B} 11 \mathrm{PE} \text { atrazine } 0.5 \mathrm{~kg} \mathrm{ha}^{-1}+ \\
\mathrm{HW}+\mathrm{IC}\end{array}$ & $\begin{array}{l}8.10 \\
(63.62)\end{array}$ & $\begin{array}{l}9.36 \\
(85.67)\end{array}$ & $\begin{array}{l}7.47 \\
(53.85)\end{array}$ \\
\hline $\mathrm{T}_{11}-30 \mathrm{~B} 11 \mathrm{No} \mathrm{WC}$ and only IC & $15.74(245.85)$ & $\begin{array}{l}15.13 \\
(226.78)\end{array}$ & $\begin{array}{l}14.97 \\
(222.00)\end{array}$ \\
\hline $\mathrm{T}_{12}-30 \mathrm{~B} 11$ No $\mathrm{WC}$ and no IC & $17.12(291.03)$ & $\begin{array}{l}15.91 \\
(251.15)\end{array}$ & $\begin{array}{l}16.06 \\
(255.96)\end{array}$ \\
\hline $\begin{array}{l}\mathrm{T}_{13}-\mathrm{BIO} 9681 \mathrm{PE} \text { atrazine } 0.5 \mathrm{~kg} \mathrm{ha}^{-1}+ \\
\text { HW+ IC }\end{array}$ & $\begin{array}{l}7.95 \\
(61.21)\end{array}$ & $\begin{array}{l}8.84 \\
(76.16)\end{array}$ & $\begin{array}{l}6.86 \\
(45.02)\end{array}$ \\
\hline $\mathrm{T}_{14}$-BIO9681No WC and no IC & $16.56(272.3)$ & $\begin{array}{l}15.53 \\
(239.32)\end{array}$ & $\begin{array}{l}15.32 \\
(232.73)\end{array}$ \\
\hline $\begin{array}{l}\mathrm{T}_{15} \text {-CoHM5 PE atrazine } 0.5 \mathrm{~kg} \mathrm{ha}^{-1}+ \\
\text { HW+ IC }\end{array}$ & $\begin{array}{l}8.49 \\
(70.03)\end{array}$ & $\begin{array}{l}9.82 \\
(94.53)\end{array}$ & $\begin{array}{l}7.20 \\
(49.79)\end{array}$ \\
\hline $\mathrm{T}_{16}$-CoHM5 No WC and no IC & $17.21(294.18)$ & $\begin{array}{l}17.10 \\
(290.48)\end{array}$ & $\begin{array}{l}16.98 \\
(286.30)\end{array}$ \\
\hline SEd & 1.41 & 1.10 & 1.05 \\
\hline $\mathrm{CD}(\mathrm{P}=\mathbf{0 . 0 5})$ & 2.89 & 2.26 & 2.14 \\
\hline
\end{tabular}

$\mathrm{T}_{1}-\mathrm{T}_{16}$ - Non Transgenic maize hybrids; HW-Hand weeding; IC-Insect control; WC-Weed control 
Table.4 Effect of glyphosate application on grain yield and weed control efficiency of transgenic maize

\begin{tabular}{|c|c|c|c|}
\hline \multirow[t]{3}{*}{ Treatments } & \multicolumn{3}{|c|}{ Kharif, 2010} \\
\hline & \multicolumn{2}{|c|}{$\begin{array}{r}\text { Grain yield }\left(\mathrm{t} \mathrm{ha}^{-1}\right) \\
(\%)\end{array}$} & WCE \\
\hline & $\begin{array}{c}\text { At } \\
\text { harvest }\end{array}$ & $\begin{array}{c}20 \\
\text { DAS }\end{array}$ & $\begin{array}{c}40 \\
\text { das }\end{array}$ \\
\hline $\mathrm{T}_{1}-$ T.30V92 HR Glyphosate @900 g a.e ha ${ }^{-1}$ & 11.10 & 0.00 & 98.56 \\
\hline $\mathrm{T}_{2}-\mathrm{T} .30 \mathrm{~V} 92 \mathrm{HR}$ Glyphosate @1800 g a.e ha ${ }^{-1}$ & 12.21 & 0.00 & 99.53 \\
\hline $\mathrm{T}_{3}-\mathrm{T} .30 \mathrm{~V} 92 \mathrm{HR}$ (Weedy check) & 8.84 & 0.00 & 0.00 \\
\hline T4-T.30B11HR Glyphosate @900 g a.e ha ${ }^{-1}$ & 10.97 & 0.00 & 97.72 \\
\hline $\mathrm{T}_{5}-\mathrm{T} .30 \mathrm{~B} 11 \mathrm{HR}$ Glyphosate @1800 g a.e ha ${ }^{-1}$ & 11.98 & 0.00 & 98.97 \\
\hline $\mathrm{T}_{6}-\mathrm{T} .30 \mathrm{~B} 11 \mathrm{HR}$ (Weedy check) & 9.12 & 0.00 & 0.00 \\
\hline $\begin{array}{l}\mathrm{T}_{7}-\mathrm{N} . \mathrm{T} .30 \mathrm{~V} 92 \mathrm{PE} \text { atrazine } 0.5 \mathrm{~kg} \mathrm{ha}^{-1}+ \\
\text { HW+ IC }\end{array}$ & 10.23 & 80.28 & 72.57 \\
\hline $\mathrm{T}_{8}$ - N.T.30V92 No WC and only IC & 8.33 & 0.00 & 14.66 \\
\hline $\mathrm{T}_{9}-\mathrm{N} . \mathrm{T} .30 \mathrm{~V} 92 \mathrm{No} \mathrm{WC}$ and no IC & 7.52 & 0.00 & 0.00 \\
\hline $\begin{array}{l}\text { T }_{10} \text { N.T.30B11 PE atrazine } 0.5 \mathrm{~kg} \mathrm{ha}^{-1}+ \\
\text { HW+ IC }\end{array}$ & 9.76 & 79.66 & 70.33 \\
\hline $\mathrm{T}_{11^{-}}$N.T.30B11No WC and only IC & 8.20 & 0.00 & 11.92 \\
\hline $\mathrm{T}_{12}-$ N.T.30B11 No WC and no IC & 7.35 & 0.00 & 0.00 \\
\hline $\begin{array}{l}\mathrm{T}_{13}-\mathrm{BIO} 9681 \mathrm{PE} \text { atrazine } 0.5 \mathrm{~kg} \mathrm{ha}^{-1}+\mathrm{HW}+ \\
\text { IC }\end{array}$ & 8.00 & 77.27 & 68.73 \\
\hline $\mathrm{T}_{14}-\mathrm{BIO} 9681 \mathrm{No} \mathrm{WC}$ and no IC & 6.12 & 0.00 & 0.00 \\
\hline $\begin{array}{l}\mathrm{T}_{15}-\mathrm{CoHM} 5 \mathrm{PE} \text { atrazine } 0.5 \mathrm{~kg} \mathrm{ha}^{-1}+\mathrm{HW}+ \\
\text { IC }\end{array}$ & 7.33 & 79.28 & 68.56 \\
\hline $\mathrm{T}_{16}-\mathrm{CoHM} 5 \mathrm{No} \mathrm{WC}$ and no IC & 5.08 & 0.00 & 0.00 \\
\hline SEd & 0.41 & - & - \\
\hline $\mathrm{CD}(\mathrm{P}=0.05)$ & 0.84 & - & - \\
\hline
\end{tabular}


Table.5 Effect of glyphosate application on grain yield, weed index and weed control efficiency of non transgenic maize

\begin{tabular}{|c|c|c|c|}
\hline \multirow[t]{2}{*}{ Treatments } & \multicolumn{3}{|c|}{$\begin{array}{c}\text { Kharif, } 2011 \\
\text { Grain yield }\left(\mathrm{t} \text { ha }^{-1}\right) \text { WCE }(\%)\end{array}$} \\
\hline & At harvest & 20 DAS & 40 DAS \\
\hline $\begin{array}{c}\begin{array}{l}\text { T} \\
1\end{array} \text {-N.T.30V92 POE Glyphosate @ } 900 \text { g a.e } \\
\text { ha }^{-1}\end{array}$ & 9.12 & 5.14 & 96.15 \\
\hline $\begin{array}{l}\mathrm{T}_{2}-\text { N.T.30V92 POE Glyphosate @ } 1350 \mathrm{~g} \\
\text { a.e ha-1 }\end{array}$ & 10.36 & 14.29 & 97.66 \\
\hline $\begin{array}{l}\mathrm{T}_{3} \text {-N.T.30V92 POE Glyphosate @ } 1800 \mathrm{~g} \\
\text { a.e ha }\end{array}$ & 11.23 & 8.73 & 99.14 \\
\hline $\begin{array}{c}\begin{array}{c}\text { T} \\
4\end{array}-\text { N.T.30B11 POE Glyphosate @ } 900 \text { g a.e } \\
\text { ha }^{-1}\end{array}$ & 8.25 & 21.41 & 95.86 \\
\hline $\begin{array}{l}\text { T5-N.T.30B11 POE Glyphosate @ } 1350 \text { g a.e } \\
\text { ha }^{-1}\end{array}$ & 9.52 & 14.16 & 97.17 \\
\hline $\begin{array}{l}\mathrm{T}_{6}-\text { N.T.30B11 POE Glyphosate @ } 1800 \mathrm{~g} \\
\text { a.e ha }\end{array}$ & 10.39 & 11.15 & 98.87 \\
\hline $\mathrm{T}_{7}-30 \mathrm{~V} 92 \mathrm{PE}$ atrazine $0.5 \mathrm{~kg} \mathrm{ha}^{-1}+\mathrm{HW}+\mathrm{IC}$ & 8.72 & 82.26 & 68.96 \\
\hline $\mathrm{T}_{8}-30 \mathrm{~V} 92 \mathrm{No} \mathrm{WC}$ and only IC & 7.40 & 13.97 & 10.25 \\
\hline $\mathrm{T}_{9}-30 \mathrm{~V} 92 \mathrm{No} \mathrm{WC}$ and no IC & 6.20 & 0.00 & 0.00 \\
\hline $\begin{array}{l}\mathrm{T}_{10}-30 \mathrm{~B} 11 \mathrm{PE} \text { atrazine } 0.5 \mathrm{~kg} \mathrm{ha}^{-1}+\mathrm{HW}+ \\
\text { IC }\end{array}$ & 8.01 & 80.03 & 65.71 \\
\hline $\mathrm{T}_{11}-30 \mathrm{~B} 11 \mathrm{No} \mathrm{WC}$ and only IC & 6.80 & 13.57 & 8.31 \\
\hline $\mathrm{T}_{12}-30 \mathrm{~B} 11 \mathrm{No} \mathrm{WC}$ and no IC & 6.22 & 0.00 & 0.00 \\
\hline $\begin{array}{l}\mathrm{T}_{13}-\mathrm{BIO} 9681 \mathrm{PE} \text { atrazine } 0.5 \mathrm{~kg} \mathrm{ha}^{-1}+\mathrm{HW}+ \\
\text { IC }\end{array}$ & 7.10 & 78.97 & 63.82 \\
\hline $\mathrm{T}_{14}$-BIO9681No WC and no IC & 5.60 & 0.00 & 0.00 \\
\hline $\begin{array}{l}\mathrm{T}_{15}-\mathrm{CoHM} 5 \mathrm{PE} \text { atrazine } 0.5 \mathrm{~kg} \mathrm{ha}^{-1}+\mathrm{HW}+ \\
\text { IC }\end{array}$ & 6.10 & 73.19 & 61.68 \\
\hline $\mathrm{T}_{16}$-CoHM5 No WC and no IC & 4.80 & 0.00 & 0.00 \\
\hline SEd & 0.80 & - & - \\
\hline $\mathrm{CD}(\mathrm{P}=0.05)$ & 1.64 & - & - \\
\hline
\end{tabular}

$\mathrm{T}_{1}-\mathrm{T}_{16}$ - Non Transgenic maize hybrids., HW-Hand weeding; IC-Insect control; WC-Weed control.

Different rates of glyphosate under transgenic maize hybrids recorded more than 90 per cent control efficiency at 40 DAS. Whereas, at the same time PE application of atrazine in nontransgenic hybrids recorded only 70 to 80 per cent. This might be due to application of glyphosate which did not allow weeds to accumulate sufficient biomass and ultimately resulted in higher weed control efficiency. Properly timed sequential application of glyphosate was effective in season-long control of common waterhemp (Amaranthus rudis), giant foxtail (Setaria faberi), velvetleaf (Abutiliontheophrasti), common cocklebur (Xanthum strumarium) and common lamb squarters (Chenopodium album) at levels more than 90 per cent through the season was reported by (Hellwig et al., 2002). 
In conclusion the results of this experiment indicated that, lesser weed density and higher weed control efficiency were achieved with post emergence application of glyphosate at $1800 \mathrm{~g}$ a.e $\mathrm{ha}^{-1}$ in transgenic and post emergence controlled application of glyphosate at $1800 \mathrm{~g}$ a.e $\mathrm{ha}^{-1}$ in non transgenic hybrid of 30V92 during kharif2010and kharif 2011 seasons, respectively. Enhanced complete control of broad spectrum of weeds promotes higher productivity and profitability with higher grain yield during both the kharif seasons.

\section{References}

Ferrel, J.A. and W.W. Witt. 2002. Comparison of glyphosate with other herbicides for weed control in corn (Zea mays): efficacy and economics. Weed Technol., 16: 701-706.

Hellwig, K.B., Johnson, W.G. and Scharf, P.C. 2002. Grassweeds interference and nitrogen accumulation in no tillage corn. Weed Sci., 50: 757-762.

Malviya, A. and Singh. B. 2007. Weed dynamics, productivity and economics of maize (Zea mays) as affected by integrated weed management under rainfed condition. Indian J. Agron., 52:321- 324.

Mundra, S.L., Vyas, A.K. and Maliwal, P.L. 2003. Effect of weed and nutrient management on weed growth and productivity of maize (Zea mays L.). Indian J. Weed Sci., 35(1\&2): 57-61.

Murphy, S.D., Yakuba, Y., Weise, S.F. and Swanton, C.J. 1996. Effect of planting patterns on inter row and competition between corn and late emerging weeds. Weed Sci., 44: 865-87.

Nadeem, M.A., Ahmad, R., Khalid, M., Naveed, M., Tanveer, A and Ahmad, J.N. 2008. Growth and yield response of autumn planted maize (Zea mays) and its weeds to reduced doses of herbicide application in combination with urea. Pak. J. Bot., 40(2): 667676.

Norsworthy, J.K., Burgos, N.R. and Oliver, L.R. 2001. Differences in weed tolerance to glyphosate involve different mechanisms. Weed Technol., 15: 725-731.

Summons, R.D., Grugs, K.J., Andersen, K.S., Johnson, K.A. and Sikors, J.A. 1995. Re-evaluating glyphosate as a transition-state inhibitor of EPSP synthase: Identification of an EPSP synthase-EPSP glyphosate ternary complex. Biochemistry., 34: 64336440.

Suwunnamek, U. and Parker, C. 1975. Control of Cyperus rotundus with glyphosate, The influence of ammonium sulfate and other additives. Weed Res., 15: 13-19.

Tharp, B.E., Schabenberger, O and Kells, J.J. 1999. Response of annual weed species to glufosinate and glyphosate. Weed Technol., 13: 542-547.

Wilcut, J.W., Coble, H.D., York, A.C. and Monks, D. W. 1996. The niche for herbicide-resistant crops in U.S. Agriculture. In: Herbicide-Resistant Crops: Agricultural, Environmental, Economic, Regulatory, and Technical Aspects, S.O. Duke (ed.) CRC Press, Boca Raton, FL. pp. 213-230.

\section{How to cite this article:}

Sivagamy, K., C. Chinnusamy and Parasuraman, P. 2019. Influence of Post Emergence Application of K Salt of Glyphosate on Weed Control and Yield of Transgenic Stacked and Non Transgenic Maize Hybrids in Summer Irrigated Eco systems of Tamil Nadu, India. Int.J.Curr.Microbiol.App.Sci. 8(01): 1275-1284. doi: https://doi.org/10.20546/ijcmas.2019.801.135 